\title{
The Expression of miR-155-5p and Local Matrix Gla Protein in Meningiomas
}

\author{
Simona Roxana Gheorghe ${ }^{1 *}$, Cătălin Marian², Ligia Gabriela Tătăranu³, Anica \\ Dricu $^{4}$, Cees Vermeer ${ }^{5}$, Ciprian Nicolae Silaghi ${ }^{1}$, Alexandra Marioara Crăciun ${ }^{1}$
}

1. Department of Medical Biochemistry, “Iuliu Hațieganu” University of Medicine and Pharmacy, Romania

2. Department of Biochemistry and Pharmacology, "Victor Babeş" University of Medicine and Pharmacy, Romania

3. Department of Neurosurgery, "Bagdasar-Arseni” Clinical Emergency Hospital, Romania 4. Department of Biochemistry, University of Medicine and Pharmacy of Craiova, Romania

5. Cardiovascular Research Institute CARIM, Maastricht University, Netherlands

\begin{abstract}
Meningiomas are classified by the World Health Organization (WHO) in three grades, based on morphological features. Independent of this grading, the presence of calcification in meningiomas influences their growth rate. The messenger RNA of matrix Gla protein (MGP), an extra-hepatic protein with different conformations involved in the homeostasis of ectopic calcification has been found in meningiomas and was shown to be regulated in breast cancer by miR-155-5p, a specific micro RNA. Therefore, we investigated the expression of miR-155-5p and its relationship with local MGP conformations in different grade meningiomas. According to the WHO classification, our 41 samples of meningiomas were stratified in groups WHO I and WHO II. Using real time polymerase chain reaction, we observed a higher miR-155-5p expression in group WHO I versus group WHO II [with a fold change (FC) of $3.83, p=0.027)]$. Moreover, the expression of miR-155-5p was higher in calcified tumors compared to non-calcified tumors in all samples $(F C=3.01, p=0.047)$ and in group WHO I $(F C=3.65, p=0.048)$. Utilizing immunohistochemistry, we determined the concurrent presence of all MGP conformations in calcified meningiomas. This study was the first to establish higher miR-155-5p expression in grade WHO I and calcified meningiomas, which could
\end{abstract}

\footnotetext{
* Corresponding author: Simona Roxana Gheorghe, Department of Medical Biochemistry, "Iuliu Hațieganu” University of Medicine and Pharmacy, Romania. E-mail: simi_gheorghe@yahoo.com
} 
improve molecular classification and targeted therapy and also the presence of all MGP conformations in calcified meningiomas, confirming the existence of an anti-calcification mechanism in meningiomas.

Keywords: histology, biochemistry, molecular biology, experimental laboratory and clinical studies

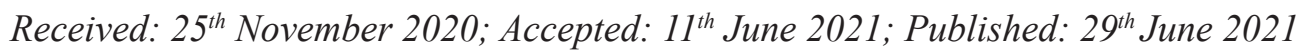

\section{Introduction}

Meningiomas are the most common primary tumors of the central nervous system, often accidentally diagnosed (1). Originating from the arachnoid cap cells found in the meninges and with a pathognomonic histology represented by whorls of meningothelial cells (2), they can develop in various locations. The symptomatology is usually nonspecific and greatly dependent on the location of the tumor. The World Health Organization (WHO) has classified meningiomas into three grades based on their histological characteristics (3). The majority are grade I (benign), representing $80 \%$, approximately $17 \%$ are grade II (atypical), while grade III (anaplastic) meningiomas account for a very small percentage of the cases (4). Grade II and III meningiomas have an unpredictable outcome due to their aggressive behavior, while grade I tumors are more idle (5). It was also demonstrated that calcified meningiomas, regardless of their WHO grade, have a lower growth rate, while the absence of calcification was associated with rapid tumor growth (6). An important extra-hepatic vitamin K dependent protein, matrix Gla protein (MGP) is involved in the inhibitory mechanism of ectopic calcification (7) and can present different conformations: uncarboxylated MGP (ucMGP), carboxylated MGP (cMGP), desphosphorylated MGP (dpMGP), phosphorylated MGP (pMGP), or combinations thereof. To the best of our knowledge, only one study (8) has been published regarding MGP in meningiomas, more specifically, it reported the expression of MGP mRNA in the vascular smooth muscle cells of the blood vessels of the tumor, but the local presence of the protein was not assessed.
A previous study investigating the regulation of MGP in breast cancer found that MGP was significantly influenced by a specific micro RNA (miRNA), identified as miR-155-5p, leading to cell proliferation and invasiveness (9). miRNAs are short noncoding RNA molecules consisting of approximately 22 nucleotides which modulate several paramount biological processes, such as proliferation, differentiation and apoptosis, as well as tumor development and metastasis (10). Out of 200 miRNAs analyzed, miR-155-5p and 13 other miRNAs were labeled as the meningioma miRNA "fingerprint" because of their significantly different expression compared with normal adjacent tissue (11).

Based on the existing literature which reported the expression of MGP mRNA and miR-155$5 \mathrm{p}$ in meningiomas and the down-regulation of MGP by miR-155-5p in breast cancer, our purpose was to assess the presence of all MGP conformations and to determine the expression of miR-155-5p in calcified/non-calcified and different WHO grade meningiomas, as well as to establish whether miR-155-5p regulates the local presence of MGP in these tumors.

Therefore, we conducted the first pilot study to establish whether miR-155-5p chaperones the local presence of MGP in meningiomas.

\section{Materials and Methods}

Samples of tumor tissue from 41 patients undergoing surgery for tumor resection at the "Bagdasar-Arseni" Clinical Emergency Hospital were collected between 2006 and 2012 while demographic information was obtained from patient records. We enrolled subjects with a postopera- 
tive histopathological diagnosis of meningioma who did not undergo treatment with vitamin $\mathrm{K}$ antagonists. The histopathological grading was performed following the WHO classification (3). Subjects diagnosed with grade I meningioma were included in group WHO I, while those with grade II meningioma were assigned to group WHO II. Additionally, regardless of the WHO grade, the subjects were divided in calcified and non-calcified groups based on the presence or absence of tumor calcification. The study was conducted according to the guidelines enclosed in the Declaration of Helsinki and all procedures involving human subjects were approved by the Medical Ethics Committee of "Iuliu Hațieganu" University of Medicine and Pharmacy. Written informed consent was obtained from all subjects before the enrollment in the study.

\section{Immunohistochemical tissue staining and evaluation}

From the paraffin embedded tissue sample blocks, with the help of a microtome, $4 \mu \mathrm{m}$ thick sections were cut and mounted on glass slides. Next, after tissue deparaffinization and rehydration, the slides were stained with hematoxylin-eosin. Each slide was examined and evaluated by a pathology specialist identifying the following types of meningiomas: meningothelial, transitional, and atypical.

For the immunohistochemical staining of the MGP conformations, we used specific monoclonal antibodies against ucMGP (residues 3549), cMGP (residues 35-54), dpMGP (residues 3-15), and pMGP (residues 3-15) provided by VitaK BV (Maastricht, The Netherlands). After tissue rehydration, samples were heated in a $0.2 \%$ citrate bath for antigen retrieval and incubated with diluted antibodies against ucMGP $(0.9 \mu \mathrm{g} / \mathrm{ml}), \mathrm{cMGP}(1.0 \mu \mathrm{g} / \mathrm{ml}), \mathrm{dpMGP}(1.0 \mu \mathrm{g} /$ $\mathrm{ml})$, or pMGP $(0.75 \mu \mathrm{g} / \mathrm{ml})$. The specific antibodies were diluted in blocking reagent (Roche Diagnostics, Germany). After adding the pri- mary antibody to the slides, they were incubated overnight at $4^{\circ} \mathrm{C}$. The following day, horse radish peroxidase-conjugated rabbit anti-mouse IgG (Dako, Denmark), diluted 1:100 was used as the secondary antibody. We used NovaRED substrate kit (Vector Laboratories, USA) in order to expose the antibodies. For the cell nuclei staining, we used hematoxylin and preserved the samples with coverslips mounted with Entellan (Merck, Germany). Negative controls were obtained by omitting the primary antibody.

For the identification of calcification, we used the von Kossa staining by which the sample slides were deparaffinized and rehydrated, followed by 5 minutes incubation with $1 \%$ silver nitrate. After washing, we applied sodium thiosulfate and sodium formaldehyde for 1 minute to eliminate the excess of silver nitrate. We used nuclear fast red as a counterstain and covered the samples with coverslips.

Sample slides were evaluated by a specialist blinded to the sample and subject information. Staining pattern for MGP conformations and von Kossa was defined as: negative - absence of staining throughout the sample, and positive presence of staining in at least one microscopic field.

\section{miR-155-5p detection process}

Total RNA was extracted from the 41 tissue samples mounted on slides after xylene deparaffinization using the miRNeasy FFPE kit (Qiagen) according to manufacturer protocol. The total RNA was eluted in $14 \mu \mathrm{L}$ RNAse/DNAse free water. Quantitation was performed with the Qubit 2.0 Fluorometer (ThermoFisher) with the RNA BR Assay Kit (ThermoFisher). The mean RNA concentration was $48 \mathrm{ng} / \mu \mathrm{L}$ (range $1-454$ $\mathrm{ng} / \mu \mathrm{L}$ ). cDNA synthesis for specific miRNA detection was carried out using miScript II RT kit (Qiagen) and qPCR reactions were performed in triplicate on an ABI 7900 HT real time PCR machine (ThermoFisher), using the miScript 
Sybr green PCR kit (Qiagen) and miScript Primer assays (Qiagen) for miR-155-5p and RNU6 as housekeeping gene. An initial 15 min polymerase activation step was followed by 40 cycles $\left(94^{\circ} \mathrm{C} / 15 \mathrm{~s}, 55^{\circ} \mathrm{C} / 30 \mathrm{~s}, 70{ }^{\circ} \mathrm{C} / 35 \mathrm{~s}\right)$. The miR-155-5p expression was calculated using the comparative $\Delta \mathrm{Ct}$ method relative to the RNU6 (12). The fold change (FC) was calculated based on the following formula: $\mathrm{FC}=2^{(\Delta \mathrm{Ct} \text { group } 2-\Delta \mathrm{Ct} \text { group }}$ 1).

\section{Statistical analysis}

For statistical analysis we used SPSS 15.0 (SPSS, Chicago, USA) and statistical significance was considered on two-tailed tests at $\mathrm{p}$ values $<0.05$. The distribution of continuous variables was assessed with the Kolmogorov-Smirnoff test. The variables were normally distributed and were expressed as mean \pm standard deviation (SD). We reported the Spearman's Rho coefficient for the association between nominal variables. Student $\mathrm{t}$-test was used to assess the differences between groups.

\section{Results}

The study group included a total of 41 subjects, 18 males and 23 females with the average age of $53 \pm 13$ (mean \pm SD). Based on the histopathology of the tumor tissue, we diagnosed three types of meningiomas: meningothelial, transitional, and atypical. According to the WHO classification, the first two are considered grade I meningio- mas, while the latter is a grade II meningioma. Taking into consideration this classification, we comprised two study groups: WHO I $(n=29)$ and WHO II $(n=12)$. Regarding the presence of local MGP, out of all 41 samples, 22 were positive for all protein conformations, 16 belonging to $\mathrm{WHO}$ I and 6 to WHO II. The specific immunohistochemical staining for all MGP conformations was consistent with the presence of calcification in the tumors.

When analyzing all the samples $(n=41)$, there was a significant difference in $\Delta \mathrm{Ct}$ in group WHO I versus group WHO II (5.77 \pm 2.55 vs. $7.71 \pm 2.1)$, with a FC of $3.837(\mathrm{p}=0.027)$ in miR$155-5 \mathrm{p}$ expression between the two groups.

Furthermore, we divided the study group into tumors with calcification and tumors without calcification, regardless of their WHO grade, as shown in Table 1.

The analysis of the immunohistochemical samples revealed that all calcified tumors were positive for all MGP conformations. The specific staining for MGP was observed to be extracellular, around the calcified regions, bordering or coating the mineral accumulations. A caption showing all MGP conformations around the calcified area is presented in Figure 1. The non-calcified samples were negative for any MGP conformations.

Finally, we performed correlations between $\Delta \mathrm{Ct}$, WHO classification, and the presence of local MGP conformations. We found a positive correlation between miR-155-5p expression and

Table 1. $\Delta \mathrm{Ct}$ values and FC of calcified versus non-calcified samples.

\begin{tabular}{ccccccc}
\hline & \multicolumn{2}{c}{ All } & \multicolumn{2}{c}{ WHO I } & \multicolumn{2}{c}{ WHO II } \\
$\mathrm{n}=41$ & \multicolumn{2}{c}{$\mathrm{n}=29$} & \multicolumn{2}{c}{$\mathrm{n}=12$} \\
\hline & $\begin{array}{c}\text { Calcified } \\
\mathrm{n}=22\end{array}$ & $\begin{array}{c}\text { Non-calcified } \\
\mathrm{n}=19\end{array}$ & $\begin{array}{c}\text { Calcified } \\
\mathrm{n}=16\end{array}$ & $\begin{array}{c}\text { Non-calcified } \\
\mathrm{n}=13\end{array}$ & $\begin{array}{c}\text { Calcified } \\
\mathrm{n}=6\end{array}$ & $\begin{array}{c}\text { Non-Calcified } \\
\mathrm{n}=6\end{array}$ \\
\hline $\boldsymbol{\Delta}$ & $5.60 \pm 2.32$ & $7.19 \pm 2.65$ & $4.93 \pm 2.22$ & $6.80 \pm 2.63$ & $7.37 \pm 1.64$ & $8.05 \pm 2.74$ \\
\hline FC(p-value) & $3.01(0.047)$ & \multicolumn{2}{c}{$3.65(0.048)$} & $1.6(0.615)$ \\
\hline
\end{tabular}

The data are presented as mean \pm SD or number, as appropriate. Abbreviations: ucMGP, uncarboxylated matrix gla protein; cMGP, carboxylated matrix gla protein; dpMGP, desphospho matrix gla protein; pMGP, phosphorylated matrix gla protein; $\Delta \mathrm{Ct}$, delta cycle threshold; FC, fold change. 


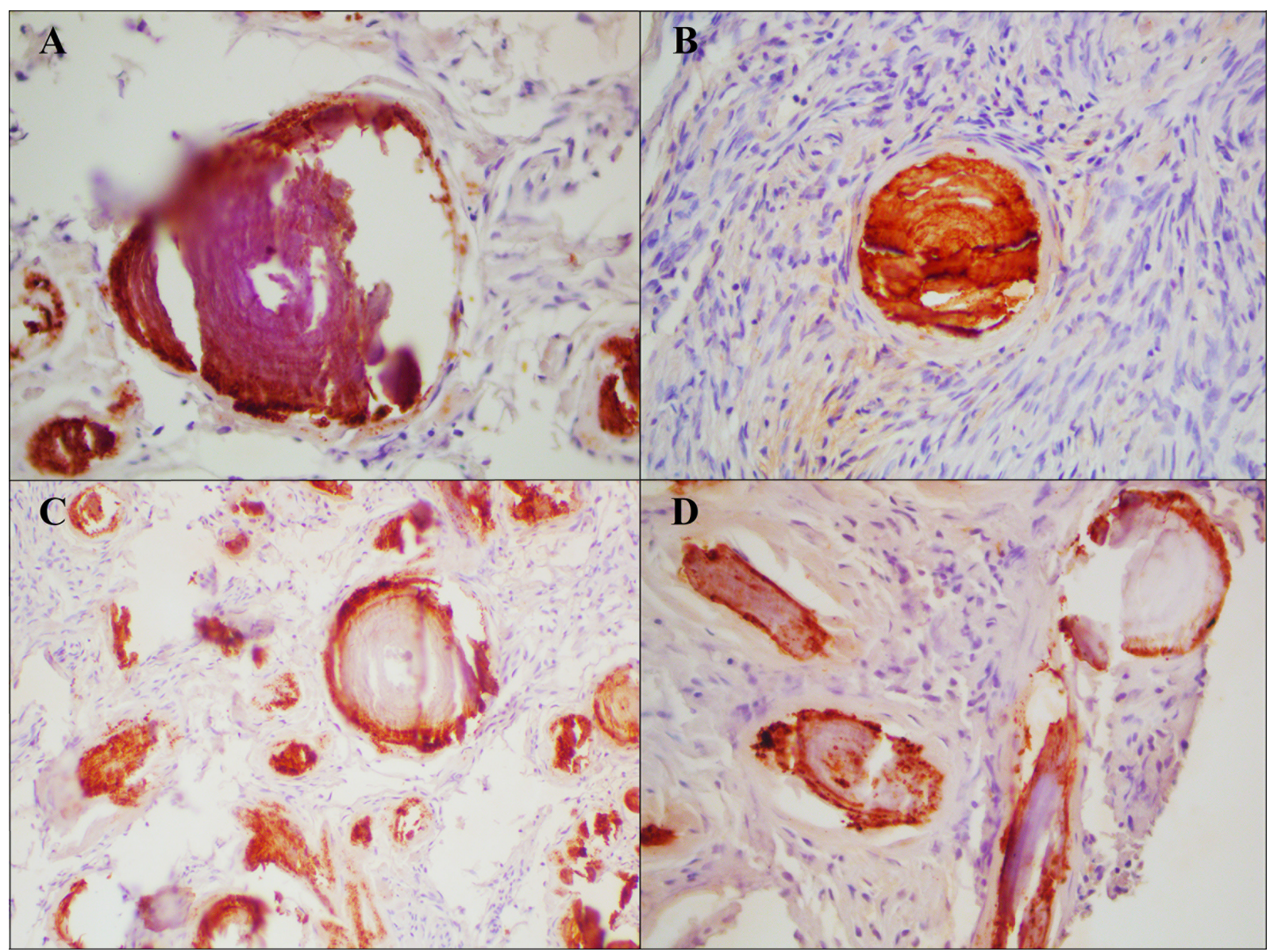

Fig. 1. Immunohistochemical images of MGP conformations in calcified meningiomas: A - ucMGP; B cMGP; C - dpMGP; D - pMGP. The local MGP conformations are represented as dark red extracellular deposits bordering or coating the calcifications; magnification $\mathbf{x} 400$ for all images.

WHO classification $(r=0.344, p=0.027)$, but miR-155-5p did not correlate with the local deposits of MGP conformations $(\mathrm{r}=-0.289, \mathrm{p}=$ 0.067).

\section{Discussion}

Our study was the first to establish an increased expression of miR-155-5p in WHO grade I and calcified meningiomas compared with WHO grade II and non-calcified tumors. Furthermore, it was the first study to identify the local presence of all MGP conformations in calcified me- ningiomas. All MGP conformations were present concurrently in the calcified meningiomas, but were absent in non-calcified meningiomas. The expression of miR-155-5p was inversely correlated with the increase in WHO grading, but no correlation was found between miR-155-5p and the presence of local MGP in meningiomas. We established a positive correlation between $\Delta \mathrm{Ct}$ and WHO classification along with a higher $\Delta \mathrm{Ct}$ in group WHO II compared with WHO I. The expression of miR-155-5p is inversely proportional to $\Delta \mathrm{Ct}$, thus the expression of miR$155-5 p$ is higher in group WHO I compared with 
WHO II. The FC is used to measure the change in the expression level of a gene and we found that miR-155-5p is almost 4 times more expressed in the tumors pertaining to grade I compared with those from grade II. The positive correlation between $\Delta \mathrm{Ct}$ and WHO classification strengthens the observation that $\Delta \mathrm{Ct}$ increases with grading, resulting in a decrease in miR-155-5p expression as the grade of the meningioma increases.

Additional to the previous study (11) reporting the overexpression of miR-155-5p in meningiomas, our research was able to determine that miR-155-5p expression is different when the tumors are divided according to the tumor grade. The higher expression of miR-155-5p in grade I meningiomas can contribute to the slow growing rate of these tumors compared with grade II where miR-155-5p expression is decreased, as it was shown that the up-regulation of miR-155-5p in head and neck tumors leads to the repression of tumor growth (13). We could hypothesize that up-regulating miR-155-5p expression in patients with grade II meningioma, could decelerate the growth of the tumor which is known to have a more aggressive behavior. Confirmation of this hypothesis requires follow-up studies on a larger number of subjects in order to introduce miR$155-5 p$ in the molecular grading of meningiomas, as well as in the molecular targeted therapy of grade II tumors.

Moreover, we found a significantly higher $\Delta \mathrm{Ct}$ in tumors without calcification compared with those with calcification in all patients. Due to the inverse proportionality between $\Delta \mathrm{Ct}$ and miR$155-5 p$, we can state that tumors with calcification have a higher expression of miR-155-5p compared with tumors without calcification. The same observation was made when analyzing calcified versus non-calcified tumors within group WHO I. In both situations, the expression of miR-155-5p was approximately 3 times higher in calcified tumors. Although the sample size of group WHO II was small $(\mathrm{n}=12)$ and the differ- ence in $\Delta \mathrm{Ct}$ was not statistically significant, we observed the same pattern of miR-155-5p expression as in overall samples and group WHO I, as well. The higher expression of miR-155-5p could be a factor in hindering the growth of calcified meningiomas, as it was demonstrated that calcified meningiomas have a slower growth rate compared with the non-calcified entities (6).

In our previous study (14), we were able to identify local accumulations of both ucMGP and cMGP around the calcification sites in all calcified meningiomas, while the non-calcified meningiomas were negative for the MGP immunohistochemical staining. The present study confirms the previous findings and, additionally, demonstrates that dpMGP and pMGP are also present at the border of calcified regions in all calcified meningiomas and absent in those without calcifications, regardless of the meningioma grade. Our observations were also consistent with the findings of Spronk et al. (15) which identified local MGP around ectopic calcification in arteries, although, at that time, it was impossible to differentiate between the conformations of MGP bordering the calcification areas.

The presence of cMGP and pMGP in calcified meningiomas could be explained by the increased excretion of cMGP to inhibit the development of the calcification by binding to the calcium crystals while the phosphorylation facilitates the protein excretion in the extracellular matrix $(16,17)$. The presence of the inactive forms, ucMGP and dpMGP could be explained by an increased rate of synthesis and subsequent extracellular secretion at a higher rate than posttranslational reactions.

Although there is a previous study showing the presence of MGP mARN in meningioma (8), this is the first study to report the local expression of the protein around the calcified regions of this tumor. The presence of MGP in meningiomas certifies that meningiomas possess calcification inhibitory mechanisms. 
We did not find any correlation between miR155-5p and local MGP deposits. However, we observed that miR-155-5p has a higher expression in calcified tumors which are consequently positive for local MGP conformations. This finding is in contrast with the previous study (9) which reported that miR-155-5p suppresses MGP in breast cancer cells. Nonetheless, a few miRNAs have been shown to switch from a down regulatory role to one of activation of their targeted molecules (18). Therefore, in calcified meningiomas, an overexpression of miR-155-5p could up-regulate local MGP excretion in order to minimize the effects of the ongoing calcification process and to inhibit the prospective mineral accumulation.

Our current study should be interpreted within the context of its possible limitations. The sample size, notably of the grade II meningiomas, was small, whereas grade III meningiomas were not enrolled in the study. For future studies, to assess the influence of miR-155-5p expression on circulating levels of MGP could be of great interest, as well as of miR-155-5p and MGP in grade III malignant meningiomas.

In our study, we established a higher miR-155$5 \mathrm{p}$ expression in grade I versus grade II and in calcified versus non-calcified menigiomas, additionally confirming the presence of MGP at the tumor level, while evaluating the relationship between mir-155-5p and local MGP conformations in meningiomas.

\section{Conclusions}

This is the first study showing that the expression of miR-155-5p was higher in WHO grade I and calcified meningiomas compared with WHO grade II and non-calcified meningiomas. All MGP conformations were present in calcified tumors and absent in non-calcified tumors, suggesting the existence of a suppressive mechanism of ectopic calcification in meningiomas. Although miR-155-5p did not correlate with the local deposits of MGP identified by immunohistochemistry, we found a positive correlation between miR-155-5p expression and WHO classification. This study could be a starting point in introducing miR-155-5p in the molecular classification of meningiomas.

\author{
Abbreviations \\ BMP-2: Bone morphogenetic protein-2 \\ cMGP: Carboxylated matrix Gla protein \\ dpMGP: Desphosphorylated matrix Gla protein \\ $\Delta \mathrm{Ct}$ : Delta cycle threshold \\ FC: Fold change \\ MGP: Matrix Gla protein \\ mRNA: messenger RNA \\ miRNA: Micro RNA \\ pMGP: Phosphorylated matrix Gla protein \\ ucMGP: Uncarboxylated matrix Gla protein \\ WHO: World Health Organization.
}

\section{Author Contributions}

SRG - Conceptualization; Investigation; Writing-original draft preparation

CM - Formal Analysis; Investigation; Writing review and editing

LGT - Investigation; Resources; Writing - review and editing

AD - Investigation; Resources; Writing - review and editing

CV - Resources; Supervision; Writing - review and editing

SNC - Formal Analysis; Validation; Writing review and editing

AMC - Project administration; Funding acquisition; Writing - review and editing

All authors have read and agreed to the manuscript.

\section{Acknowledgments}

This work was supported by the frame of $\mathrm{Eu}-$ ropean Social Found, Human Resources Devel- 
opment Operational Program 2007-2013, project no. POSDRU/159/1.5/S/138776; grant of the Ministry of Research, Innovation and Digitization, CNCS/CCCDI - UEFISCDI, project number 2/2019 (DARKFOOD), within PNCDI III and by Grant PN-II-ID-PCE-2011-3-1041, UEFISCDI.

\section{Conflicts of Interest}

The authors declare no conflict of interest. The funders had no role in the design of the study; in the collection, analyses, or interpretation of data; in the writing of the manuscript, or in the decision to publish the results.

\section{References}

1. Fathi AR, Roelcke U. Meningioma. Curr Neurol Neurosci Rep. 2013 Apr;13(4):337. DOI: 10.1007/s11910013-0337-4

2. Buerki RA, Horbinski CM, Kruser T, Horowitz PM, James CD, Lukas RV. An overview of meningiomas. Future Oncol. 2018 Sep;14(21):2161-2177. DOI: 10.2217/fon-2018-0006

3. Louis DN, Perry A, Reifenberger G, von Deimling A, Figarella-Branger D, Cavenee WK, et al. The 2016 World Health Organization Classification of Tumors of the Central Nervous System: a summary. Acta Neuropathol. 2016 Jun;131(6):803-20. DOI: 10.1007/s00401016-1545-1

4. Ostrom QT, Gittleman H, Liao P, Vecchione-Koval T, Wolinsky Y, Kruchko C, Barnholtz-Sloan JS. CBTRUS Statistical Report: Primary brain and other central nervous system tumors diagnosed in the United States in 2010-2014. Neuro Oncol. 2017 Nov 6;19(suppl_5):v1-v88. DOI: 10.1093/neuonc/nox158

5. Pasquier D, Bijmolt S, Veninga T, Rezvoy N, Villa S, Krengli M, et al; Rare Cancer Network. Atypical and malignant meningioma: outcome and prognostic factors in 119 irradiated patients. A multicenter, retrospective study of the Rare Cancer Network. Int J Radiat Oncol Biol Phys. 2008 Aug;71(5):1388-93. DOI: 10.1016/j. ijrobp.2007.12.020

6. Lee EJ, Kim JH, Park ES, Kim YH, Lee JK, Hong SH, et al. A novel weighted scoring system for estimating the risk of rapid growth in untreated intracranial meningiomas. J Neurosurg. 2017 Nov;127(5):971-980. DOI: 10.3171/2016.9.JNS161669

7. Price PA. Gla-containing proteins of bone. Connect Tissue Res. 1989;21(1-4):51-7; discussion 57-60. DOI:

\subsection{9/03008208909049995}

8. Hirota S, Nakajima Y, Yoshimine T, Kohri K, Nomura $\mathrm{S}$, Taneda $\mathrm{M}$, et al. Expression of bone-related protein messenger RNA in human meningiomas: possible involvement of osteopontin in development of psammoma bodies. J Neuropathol Exp Neurol. 1995 Sep;54(5):698-703. DOI: 10.1097/00005072199509000-00012

9. Tiago DM, Conceição N, Caiado H, Laizé V, Cancela ML. Matrix Gla protein repression by miR-155 promotes oncogenic signals in breast cancer MCF7 cells. FEBS Lett. 2016 Apr;590(8):1234-41. DOI: 10.1002/1873-3468.12155

10. Lu TX, Rothenberg ME. MicroRNA. J Allergy Clin Immunol. 2018 Apr;141(4):1202-1207. DOI: 10.1016/j. jaci.2017.08.034

11. Zhi F, Zhou G, Wang S, Shi Y, Peng Y, Shao N, et al. A microRNA expression signature predicts meningioma recurrence. Int J Cancer. 2013 Jan ;132(1):128-36. DOI: $10.1002 /$ ijc. 27658

12. Schmittgen TD, Livak KJ. Analyzing real-time PCR data by the comparative $\mathrm{C}(\mathrm{T})$ method. Nat Protoc. 2008;3(6):1101-8. DOI: 10.1038/nprot.2008.73

13. Ali Syeda Z, Langden SSS, Munkhzul C, Lee M, Song SJ. Regulatory Mechanism of MicroRNA Expression in Cancer. Int J Mol Sci. 2020 Mar;21(5):1723. DOI: 10.3390/ijms21051723

14. Gheorghe SR, Tataru LG, Dricu A, Silaghi, CN, Vermeer C, Craciun AM. Matrix Gla Protein in meningiomas: an immunohistochemical study. Rev.Chim. 2020 Apr;71(3):425-32. DOI: 10.37358/RC.20.3.8016

15. Spronk HM, Soute BA, Schurgers LJ, Cleutjens JP, Thijssen HH, De Mey JG, Vermeer C. Matrix Gla protein accumulates at the border of regions of calcification and normal tissue in the media of the arterial vessel wall. Biochem Biophys Res Commun. 2001 Nov;289(2):485-90. DOI: 10.1006/bbrc.2001.5996

16. Price PA, Chan WS, Jolson DM, Williamson MK. The elastic lamellae of devitalized arteries calcify when incubated in serum: evidence for a serum calcification factor. Arterioscler Thromb Vasc Biol. 2006 May;26(5):107985. DOI: 10.1161/01.ATV.0000216406.44762.7c

17. Wajih N, Borras T, Xue W, Hutson SM, Wallin R. Processing and transport of matrix gamma-carboxyglutamic acid protein and bone morphogenetic protein-2 in cultured human vascular smooth muscle cells: evidence for an uptake mechanism for serum fetuin. J Biol Chem. 2004 Oct;279(41):43052-60. DOI: 10.1074/jbc. M407180200

18. Vasudevan S, Tong Y, Steitz JA. Switching from repression to activation: microRNAs can up-regulate translation. Science. 2007 Dec;318(5858):1931-4. DOI: 10.1126/science. 1149460 